\title{
COHOMOLOGY OF INFINITE ALGEBRAS
}

\author{
BY \\ ALEX ROSENBERG AND DANIEL ZELINSKY(1)
}

1. Introduction. If $A$ is an arbitrary algebra over a (commutative) field $F$, the $n$th cohomology group $H^{n}(A, N)$ of $A$ with coefficients in a twosided $A$-module $N$ was introduced by Hochschild [8]. In terms of these groups, the (cohomological) dimension of $A, F-\operatorname{dim} A$ (or just $\operatorname{dim} A$ when the base field is understood), is defined as the largest $n$ such that $H^{n}(A, N) \neq 0$ for some $N$. In Hochschild's definitions, the 1-cocycles are derivations (over $F$ ) of $A$ into $N$; the 1-coboundaries are the inner derivations; the 2-cocycles are the factor sets of $A$ into $N$; the 2-coboundaries are the split (trivial) factor sets.

The assertion $H^{2}(A, N)=0$ then signifies that every algebra extension $B$ of $N$ by $A$ (where multiplication is defined in $N$ by $N^{2}=0$ ) is cleft: $B=A_{1}+N$ (vector space direct sum) with $A_{1}$ a subalgebra isomorphic to $A$. Thus the Wedderburn Principal Theorem can be rephrased in cohomology terms as follows: If $A$ is a separable algebra of finite order, then $\operatorname{dim} A<2$ ( $A$ is absolutely segregated) $[8, \S 6]$. (Actually, the Wedderburn Theorem deals with extensions of arbitrary nilpotent algebras $N$ by $A$, but the proof quickly reduces to the case $N^{2}=0$.)

The assertion $H^{1}(A, N)=0$ means every derivation of $A$ into $N$ is inner. It can also be phrased in terms of algebra extensions: If $B=A_{1}+N=A_{2}+N$ with $N^{2}=0$ and $A_{1} \cong A_{2} \cong A$, then $A_{1}=\sigma\left(A_{2}\right)$ with $\sigma$ a quasi-inner automorphism $\left(^{2}\right)$ of $B$. Hence the Malcev Uniqueness Theorem may be translated thus: If $A$ is a separable algebra of finite order, then $\operatorname{dim} A<1$. (As in the Wedderburn Theorem, the full Malcev Theorem deals with the case $N^{k}=0$, which easily reduces to the case $N^{2}=0$.)

Some of our theorems give necessary, some sufficient, and some necessary and sufficient conditions that certain algebras have dimension $n$. Any conclusions that assert $n<2$ (or $n<1$ ) can then be interpreted as generalizations of the Wedderburn Theorem (Malcev Theorem) to more general residue-class algebras.

Recently, Cartan and Eilenberg [4] have developed a new approach to cohomology theory, including Hochschild's as a special case. Their work has provided a much more flexible computational technique which has been essential in most of our proofs. In particular, we would like to thank Professor

Presented to the Society, December 28, 1954 and September 2, 1955; received by the editors April 25, 1955.

(1) This paper was written with the partial support of the National Science Foundation.

(2) $\sigma(b)=(1-n) b(1-n)^{-1}=b-n b+b n$ for fixed $n$ in $N$. 
Eilenberg for the use of the galleys of [4] and for several helpful conversations and comments with regard to our paper.

In $\$ 2$ we give a brief sketch of the techniques we need. The rest of the paper is devoted principally to proving the following results:

1. $\operatorname{dim} A=0$ if and only if $A$ is a separable algebra of finite order.

2. Let $A$ be a locally finite, semisimple algebra with minimum condition. If $A$ has order $\aleph_{0}$, then $H^{1}(A, A \otimes A) \neq 0$ (Theorem 3).

3. If $A$ has order $\aleph_{0}$ and is locally separable, then $\operatorname{dim} A=1$ ([13] and Theorem 4).

4. If $A$ is an algebra over $K$ and $K \supset F$ then $F-\operatorname{dim} K \leqq F-\operatorname{dim} A \leqq F-\operatorname{dim} K$ $+K-\operatorname{dim} A$ (Theorem 5).

5. If $A$ is a field of transcendence degree $n \leqq \infty$ over $F$, then $\operatorname{dim} A \geqq n$ (Theorem 8).

6. If $A$ is a field of transcendence degree $n<\infty$ over $F$, then $\operatorname{dim} A=n$ if and only if $A$ is finitely separably generated (i.e., $A$ is a finite, separable, algebraic extension of a rational function field) (Theorem 10).

7. If $A$ is a finitely generated extension field of $F$ with no separable generation over $F$, then $\operatorname{dim} A=\infty$ (Theorem 9).

8. Let $A$ be an extension field of transcendence degree $n$ over $F$ which is countably generated but not finitely generated. Then $\operatorname{dim} A=n+1$ or $\infty$ according as $A$ is or is not locally separably generated ( $\left.{ }^{3}\right)$ (Corollary to Theorem 10).

Items 1,2 , and 3 are theorems about algebras of dimensions 0 and 1 . Hochschild [8, Theorem 4.1] has already proved that for an algebra $A$ of finite order, $\operatorname{dim} A=0$ is equivalent to separability. Our contribution to 1 is the fact that if $A$ has infinite order then $\operatorname{dim} A \neq 0$ (Theorem 1), i.e., $H^{1}(A, N) \neq 0$ for some $N .2$, and also Theorem 2, show that in certain cases $N$ may be taken to be $A \otimes A$. It is interesting to notice (Theorem 3) that in case $A$ is semisimple of finite order, this same special module $N=A \otimes A$ has $H^{1}(A, N)=0$, regardless of whether or not $\operatorname{dim} A=0$.

Item 3 provides the major part of the information we have on the dimension of algebraic algebras of infinite order. (Algebras of finite order and dimension $>0$ have been treated extensively by Ikeda, Nagao, and Nakayama [11] and by Eilenberg [6].) Miscellaneous extra information may be obtained for example from Theorem 5-which will guarantee that any algebra has dimension $\infty$ when its center has a subfield of dimension $\infty$-and from the results of [11] and [6] coupled with Proposition 3. We should remark that the denumerability hypothesis in 3 cannot be eliminated, for if $A$ is the direct sum of uncountably many copies of $F$ and $\tilde{A}$ is the algebra obtained by adjoining a unit to $A$, then $H^{2}(\tilde{A}, A \otimes A)=H^{2}(A, A \otimes A) \neq 0[14$, p. 316]. We conjecture that, at least when the algebra is a field, nondenumerable order implies a dimension $>1$.

$\left(^{3}\right)$ A field is locally separably generated in case every finite subset can be embedded in a finitely separably generated extension of $F$. 
Item 4 is a useful theorem on subadditivity of dimension. That the dimension is not additive is shown by counterexamples in $\$ 4$. However, 4 can be easily supplemented to show that the dimensions of the algebras of polynomials and of rational functions in $n$ indeterminates are $n$. From this we can also derive a new proof that the global dimension of this polynomial ring is $n$ as well.

The remaining theorems concern dimensions of algebras which are fields. As a matter of fact, this also gives equally extensive results on the dimension of commutative algebras with minimum condition, since such an algebra has dimension $\infty$ unless it is semisimple; but a semisimple commutative algebra is a finite direct sum of fields $A_{i}$ and has dimension equal to $\max _{i} \operatorname{dim} A_{i}$.

2. Background. We shall use the following notations consistently: $A$ and $K$ for algebras over a commutative ring $F ; A^{*}$ for the inverse of $A$ (the algebra anti-isomorphic to $A$ ); $A^{e}$ for $A \otimes A^{*}$, an algebra over $F$. Tensor products will be understood to be taken over $F$ unless otherwise indicated. All algebras and rings will be assumed to have a unit. A subring shall always contain the unit of the big ring.

Modules over rings will always be unitary, and will be left-modules unless otherwise specified. If $R$ is a ring we shall use $R_{\varepsilon}$ for the left $R$-module $R$ and $R_{d}$ for the right $R$-module $R$. If $A$ is an algebra over $F$ then two-sided $A$ modules (vector spaces over $F$ having $A$ as both left- and right-operators) are exactly the same as left $A^{\circ}$-modules with $a m=(a \otimes 1) m$ and $m a=\left(1 \otimes a^{*}\right) m$ for $a$ in $A$ and $m$ in the module. In particular $A$ is a left $A^{e}$-module in a natural way. So also is $A_{8} \otimes A_{d}$; in fact the latter is just $A^{e}$ as a left $A^{e}$-module, i.e., $\left(A^{\bullet}\right)$..

The phrases semisimple and simple shall always mean semisimple and simple with minimum condition on one-sided ideals.

If $R$ is any ring Cartan-Eilenberg [4] define the left dimension of any left $R$-module $M$ as follows: A projective resolution of $M$ is an exact sequence of $R$-modules and mappings

$$
\cdots \rightarrow X_{n} \rightarrow \cdots \rightarrow X_{1} \rightarrow X_{0} \rightarrow M \rightarrow 0
$$

with $X_{i}$ a projective module ( $M_{0}$-module)-a direct summand of a free $R$ module. (One way to obtain such a projective resolution is to write $M$ as a free $R$-module $X_{0}$ modulo a relations module $Y_{0}$, write $Y_{0}=X_{1} / Y_{1}$ with $X_{1}$ free, etc.) Then the left dimension of the $R$-module $M$, denoted by $\operatorname{ldim}_{R} M$, is the smallest $n$ such that $X_{n+1}=X_{n+2}=\cdots=0$ for some projective resolution-the length of the shortest projective resolution. It is a nonnegative integer or infinity. The maximum of $1 . \operatorname{dim}_{R} M$ as $M$ ranges over all $R$-modules is called the left global dimension of $R$ and is denoted by l.gl.dim $R$.

The dimension of an algebra $A$ is $\operatorname{ldim}_{A} e A$ and is denoted by $\operatorname{dim} A$ or $F$-dim $A$ when it is desirable to indicate the base ring $F$ (entering into $A^{e}=A \otimes_{F} A^{*}$ ). If $F$ is a field the ordinary dimension of $A$ as a vector space 
over $F$ will be referred to as the order of $A$ and will be denoted by $(A: F)$.

If $M$ and $N$ are $R$-modules the group $\operatorname{Ext}_{R}^{n}(M, N)$ is defined as the $n$th (co)homology group of the cochain complex

$$
0 \rightarrow \operatorname{Hom}_{R}(M, N) \rightarrow \operatorname{Hom}_{R}\left(X_{0}, N\right) \rightarrow \operatorname{Hom}_{R}\left(X_{1}, N\right) \rightarrow \cdots
$$

where the $X$ 's arise from a projective resolution (1) of $M$. The fundamental uniqueness of $[4, \mathrm{~V}, 3]$ asserts that Ext is independent of the particular projective resolution used. Then $1 . \operatorname{dim}_{R} M$ may be defined equivalently as the largest $n$ such that $\operatorname{Ext}_{R}^{n}(M, N) \neq 0$ for some $N$. Since for an algebra $A$, Ext $_{A^{e}}^{n}(A, N) \cong H^{n}(A, N)[4, \mathrm{IX}, 6], \operatorname{dim} A$ defined as $1 . \operatorname{dim}_{A e} A$ coincides with the definition in $\$ 1$.

At several points in the discussion we shall find it necessary to use the Hochschild definitions and terminology rather than those of [4]. We therefore recall briefly what we need: The $n$-cochains of $A$ into the two-sided $A$ module $N$ are the $F$-multilinear functions from $A$ to $N$ and the coboundary operator $\delta$ defined by

$$
\begin{gathered}
\delta f\left(x_{1}, \cdots, x_{n+1}\right)=x_{1} f\left(x_{2}, \cdots, x_{n+1}\right)+\sum(-1)^{i} f\left(x_{1}, \cdots, x_{i} x_{i+1}, \cdots, x_{n+1}\right) \\
+(-1)^{n+1} f\left(x_{1}, \cdots, x_{n}\right) x_{n+1}
\end{gathered}
$$

sends $n$-cochains into $n+1$ cochains. The $n$-cochains $f$ such that $\delta f=0$ are the $n$-cocycles and the $n$-cochains of the form $\delta g$ with $g$ an $(n-1)$-cochain are the $n$-coboundaries. Then the additive group of $n$-cocycles modulo $n$-coboundaries is $H^{n}(A, N)$.

\section{Infinite algebras.}

THEOREM 1. Let $A$ be an algebra over a commutative ring $F$ which is free as an $F$-module. (This is automatic if. $F$ is a field.) If $\operatorname{dim} A=0$, then $A$ is finitely F-generated.

Proof. The following proof was kindly pointed out to us by the referee and replaces our earlier proof which was only valid in case $F$ was a field: Let $\left\{a_{i}\right\}$ be an $F$-basis of $A$. Renumbering the basis elements if necessary, [4, IX, 7.7] shows the existence of elements $\bar{a}_{1}, \cdots, \bar{a}_{k}$ in $A$ such that

$$
\text { (i) } \sum a_{i} \bar{a}_{i}=1 \text { and (ii) } \sum a a_{i} \otimes \bar{a}_{i}=\sum a_{i} \otimes \bar{a}_{i} a \text {, }
$$

for any $a$ in $A$ (cf. also [9, Theorem 5]). Let $B$ denote the $F$-module generated by $\bar{a}_{1}, \cdots, \bar{a}_{k}$. Then if we write $a a_{i}$ as an $F$-linear combination of the $a_{i}$, (ii) shows that $\bar{a}_{i} a$ is an $F$-linear combination of the $\bar{a}_{i}$. Hence $B$ is a right ideal in $A$. Now, $a=1 \cdot a=\sum a_{i} \bar{a}_{i} a$, by (i), so that $A=\sum a_{i} B$. Thus $A$ is a finitely generated $F$-module and so, of course, possesses a finite $F$-basis.

REMARKs. 1. Using [8, Theorem 4.1] we conclude that if $F$ is a field a necessary and sufficient condition for $\operatorname{dim} A=0$ is $(A: F)<\infty$ and $A$ separable. 
2. Even if $A$ has no unit element, Theorem 1 is true. Only Hochschild's definitions apply to this case but [9, Theorem 2] asserts that one may adjoin a unit to $A$ and get a new algebra $\tilde{A}$ of the same dimension. Since $A$ is $F$-free, $\widetilde{A}=A \oplus F \cdot 1$ is also and if $A$ is finitely generated as an $F$-module, $A$ will also be.

3. If $F$ is a field, $\operatorname{dim} A=0$ is equivalent to $A^{e}$ being semisimple. Hence over a field $A \otimes A^{*}$ will be semisimple if and only if $(A: F)<\infty$ and $A$ is separable.

If $F$ is a field Theorem 1 states that if $(A: F)$ is infinite, there is an $A^{e_{-}}$ module $N$ such that $H^{1}(A, N) \neq 0$. For later applications we need information about $H^{1}$ with coefficients in a specific $A^{e}$-module. Theorems exhibiting behavior of specific coefficient modules are already known. For example, if $A$ is a semisimple subalgebra of a central simple algebra $L$ of finite order, then every derivation of $A$ into $L$ is inner; i.e. $H^{1}(A, L)=0$ [12, Chap. 5, Theorem 17]. Of course, if $A$ is separable, $H^{1}(A, N)=0$ for every $A^{e}$-module $N$, but $H^{1}$ vanishes for these specific coefficients even if $A$ is inseparable. By reduction to the classical theorem we shall prove a similar result with the $A^{\mathrm{e}}$-module $A_{s} \otimes A_{d}$ as coefficients. It is interesting that $A_{s} \otimes A_{d}$ is the same module which can be used for certain algebras of infinite order to give a nonvanishing first cohomology group (Theorems 2 and 3). To investigate $H^{1}\left(A, A_{s} \otimes A_{d}\right)$ we first study $H^{1}(A, M \otimes N)$ where $M$ and $N$ are irreducible left and right $A$-modules, respectively.

Proposition 1. Let $A$ be a simple algebra of finite order over a field $F$, let $M$ and $N$ be irreducible left and right $A$-modules respectively, and $L$ be the algebra of all $F$-linear transformations on $M$. Since $L$ contain's a subalgebra isomorphic to $A$ (the operators on $M$ induced by $A$ ), $L$ may be considered as a two-sided $A$-module. As such, $L \cong M \otimes N$. Furthermore, if $P$ and $Q$ are finitely generated left and right $A$-modules respectively, $H^{1}(A, P \otimes Q)=0$.

Proof. The dual $M^{*}$ of the $F$-space $M$ is a right $A$-module isomorphic to $N$. To verify this it is sufficient to note that $M^{*}$ is irreducible, which is an immediate consequence of the fact $\left(M^{*}: F\right)=(M: F)$. Thus $M \otimes N$ is $A^{e_{-}}$ isomorphic to $M \otimes M^{*}$. Bu't the classical $F$-isomorphism $L \cong M \otimes M^{*}$ which associates to $m_{1} \otimes m_{2}^{*}$ the mapping $m \rightarrow m_{2}^{*}(m) m_{1}$ is trivially verified to be a two-sided $L$-isomorphism and so also a two-sided $A$-isomorphism.

By the theorem mentioned above [12, Chap. 5, Theorem 17],

$$
H^{1}(A, M \otimes N)=H^{1}(A, L)=0 .
$$

Since $P$ is a finite direct sum of modules isomorphic to $M$ and $Q$ is a finite direct sum of modules isomorphic to $N$, and since $H^{n}(A, X)$ distributes over finite direct sums in the second variable, $H^{1}(A, P \otimes Q)=0$.

Proposition 2. Let $A$ be a simple algebra of finite order over a field $F$, let $P$ 
and $Q$ be finitely generated left and right $A$-modules respectively and let $C$ be the centralizer of $A$ on $P \otimes Q$ :

$$
C=\{x \in P \otimes Q \mid x a=a x \text { for all } a \text { in } A\} .
$$

Then $(C: F)(A: F)=(P: F)(Q: F)$.

Proof. If $P=M$ and $Q=N$ are as in Proposition 1 , then $C$ is $A^{e}$-(hence $F$-)isomorphic to the centralizer of $A$ in $L$. The standard theorem on centralizers of simple subalgebras of central simple algebras [1, IV, Theorem 13] then asserts that $(C: F)(A: F)=(L: F)=(M: F)(N: F)$. In general $P$ is a direct sum of $p$ copies of $M, Q$ is a direct sum of $q$ copies of $N$ and hence $P \otimes Q$ is a direct sum of $p q$ copies of $M \otimes N$. The centralizer $C$ of $A$ in $P \otimes Q$ is the direct sum of the centralizers of $A$ in the direct summands. Hence $(C: F)(A: F)$ $=p q(M: F)(N: F)=(P: F)(Q: F)$.

THEOREM 2. If $F$ is a field and the F-algebra $A$ is a union of a countably infinite tower of simple subalgebras of finite order, then $H^{1}\left(A, A_{s} \otimes A_{d}\right) \neq 0$.

Proof. Write $A=\bigcup_{i=1}^{\infty} A_{i}$ with $A_{1} \subset A_{2} \subset \cdots$, a tower of simple subalgebras of finite order. Since the tower is properly infinite, we may assume, deleting some of the $A_{i}$ 's if necessary, that $\left(A_{i+1}: F\right) \geqq 3\left(A_{i}: F\right)$; this we shall have occasion to do near the end of the proof.

We write $X=A_{\bullet} \otimes A_{d}$ and introduce on $X$ a topology with base at zero consisting of the centralizers

$$
C_{i}=\left\{x \in X \mid x a-a x=0 \text { for all } a \in A_{i}\right\} .
$$

(It can be shown that this is a Hausdorff topology but we have no need for that fact.) The brunt of the proof consists in showing $X$ is not complete. In fact, we shall exhibit a sequence $\left\{x_{i}\right\}$ with $x_{i} \in C_{i}$ but with $\sum_{i=1}^{\infty} x_{i}$ not convergent in $X$. Then the mapping

$$
a \rightarrow \Delta(a)=\sum_{i=1}^{\infty}\left(x_{i} a-a x_{i}\right)
$$

is a non-inner derivation of $A$ into $X$. This mapping is well-defined because each $a$ in $A$ is in some $A_{k}$ and since $x_{i} \in C_{i} \subset C_{k}$ for $i \geqq k$, all terms in the series (2) beyond the $(k-1)$ st vanish. This also shows that on each $A_{k}$ the mapping is a derivation; in fact, inner derivation by $y_{k}=\sum_{i=1}^{k-1} x_{i}$. Hence $\Delta$ is a derivation on $A$. However, if $\Delta$ is inner on $A$, i.e., $\Delta(a)=x a-a x$ for fixed $x \in X$, then for $a \in A_{k}, x a-a x=y_{k} a-a y_{k}, x-y_{k} \in C_{k}$, so that $x=\lim y_{k}=\sum x_{i}$, contrary to the choice of $\left\{x_{i}\right\}$. It thus remains to choose $\left\{x_{i}\right\}$.

Denote by $X_{i}$ the $A_{i}^{e}$-module $A_{i s} \otimes A_{i d}$, which gives $X=\bigcup_{i=1}^{\infty} X_{i}$. Let $U_{i}$ and $V_{i}$ be left and right $A_{i}$-complements of $A_{i}$ in $A_{i+1}$, respectively. Then

$$
X_{i+1}=X_{i} \oplus\left(A_{i s} \otimes V_{i}\right) \oplus\left(U_{i} \otimes A_{i d}\right) \oplus\left(U_{i} \otimes V_{i}\right)
$$

as $A_{i}^{e}$-modules. Denote by $C_{i}^{\prime}$ the centralizer $C_{i} \cap\left(U_{i} \otimes V_{i}\right)$ of $A_{i}$ on $U_{i} \otimes V_{i}$ 
and by $C_{i+1}^{\prime}$ the projection on $U_{i} \otimes V_{i}$ of $C_{i+1} \cap X_{i+1}$. Then by Proposition 2,

$$
\left(C_{i}^{\prime}: F\right)=\left(U_{i}: F\right)\left(V_{i}: F\right) /\left(A_{i}: F\right)=\left[\left(A_{i+1}: F\right)-\left(A_{i}: F\right)\right]^{2} /\left(A_{i}: F\right)
$$

whereas, also by Proposition 2,

$$
\left(C_{i+1}^{\prime}: F\right) \leqq\left(C_{i+1} \cap X_{i+1}: F\right)=\left(A_{i+1}: F\right)^{2} /\left(A_{i+1}: F\right)=\left(A_{i+1}: F\right) .
$$

Denoting $\left(A_{i+1}: F\right) /\left(A_{i}: F\right)$ by $t$ and assuming $t \geqq 3$, we have

$$
\left(C_{i}^{\prime}: F\right) /\left(C_{i+1}^{\prime}: F\right) \geqq(t-1)^{2} / t>1
$$

which proves that there must exist an element $x_{i}$ in $C_{i}^{\prime}$ which is not in $C_{i+1}^{\prime}$. This $x_{i}$ surely satisfies

$$
\begin{aligned}
& x_{i} \in C_{i}^{\prime} \subset X_{i+1} \cap C_{i}, \\
& x_{i} \notin X_{i}+C_{i+1} .
\end{aligned}
$$

If now $\sum_{i=1}^{\infty} x_{i}$ converges to $x$ in $X$, then for each $n$ and all sufficiently large $r$ (choose one $r \geqq n$ ), $C_{n+1}$ contains $\sum_{i=1}^{r} x_{i}-x=x_{n}+\sum_{i=1}^{n-1} x_{i}+\sum_{i=n+1}^{r} x_{i}-x$, with $\sum_{i=1}^{n-1} x_{i} \in X_{n}$ and $\sum_{i=n w 1}^{r} x_{i} \in C_{n+1}$ by (3). Thus $x_{n} \in C_{n+1}+X_{n}-x$ and if we choose $n$ so that $x \in X_{n}$ we have $x_{n} \in C_{n+1}+X_{n}$, a contradiction. This proves Theorem 2 .

THEOREM 3. Let $A$ be a locally finite $\left(^{4}\right)$, semisimple algebra over a field $F$. If $(A: F)=\boldsymbol{\aleph}_{0}$ then $H^{1}\left(A, A_{s} \otimes A_{d}\right) \neq 0$, but if $(A: F)<\boldsymbol{\aleph}_{0}$ then $H^{1}\left(A, A_{s} \otimes A_{d}\right)$ $=0$.

Proof. If $A=\sum_{\oplus} A_{i}$ is the decomposition of $A$ as a direct sum of simple algebras $A_{i}$, then by $[4, \mathrm{IX}, 5.3], H^{1}\left(A, A_{s} \otimes A_{d}\right)=\sum_{\oplus} H^{1}\left(A_{i}, A_{i s} \otimes A_{i d}\right)$. Thus we may reduce to the case where $A$ is simple.

In case $(A: F)=\boldsymbol{\aleph}_{0}$, write $A=T \otimes D$ where $T$ is a total matrix algebra over $F$ and $D$ is a division subalgebra (hence $D$ is also locally finite). Then $D$ is a union $\bigcup_{1}^{\infty} D_{i}$ of a countable tower of division subalgebras of finite order (e.g., $D_{i}$ is the algebra generated by the first $i$ elements in a basis of $D$ over $F)$. Setting $A_{i}=T \otimes D_{i}$, we have a countably infinite tower $A_{1} \subset A_{2} \subset \cdots$ of simple subalgebras of $A$ whose union is $A$. Theorem 2 is applicable and completes the proof that $H^{1}\left(A, A_{8} \otimes A_{d}\right) \neq 0$.

If $(A: F)<\aleph_{0}, H^{1}\left(A, A_{s} \otimes A_{d}\right)=0$ by Proposition 1 .

REMARK. There is no loss of generality in Theorems 2 and 3 in assuming that $F$ is a field. For if a simple ring $A$ is an algebra over a commutative ring $F$, the center of $A$ is a field containing $F \cdot 1$, hence also the quotient field $K$ of $F \cdot 1$. Thus $A$ is also an algebra over $K$. Moreover $A \otimes A^{*}=A \otimes_{K} A^{*}$. To see this, it is enough to show $k \otimes 1=1 \otimes k$ for each $k$ in $K$. But $k=f_{1} f_{2}^{-1}$ with $f_{1}, f_{2}$ in $F \cdot 1$, so that $k \otimes 1=f_{1} f_{2}^{-1} \otimes f_{2} f_{2}^{-1}=1 \otimes k$. Thus the cohomology groups

(4) Every finite subset can be embedded in a subalgebra of finite order. 
and the dimension of $A$ over $F$ are the same as over $K$. This remark could also be used to generalize the results of $\$ 5$ to algebras over commutative rings instead of over fields.

If $F$ is a field, Theorem 1 asserts that if $(A: F)$ is infinite then $\operatorname{dim} A \geqq 1$. In the next section we shall determine the exact dimension of some transcendental algebras; as the final result of the present section we include a theorem due to Kuročkin [13] which shows that certain algebraic algebras have dimension exactly 1 .

Theorem 4. If $A$ is a locally separable $\left(^{5}\right)$ algebra over a field $F$ and $(A: F)$ $=\aleph_{0}$, then $\operatorname{dim} A=1$.

Proof. The two assumptions about $A$ immediately imply that $A$ contains a denumerable tower of separable subalgebras of finite order $A_{1} \subset A_{2}$ $\subset A_{3} \subset \cdots$ such that $A=\bigcup_{1}^{\infty} A_{i}$. Since $\operatorname{dim} A_{i}=0$ Theorem 4 will follow immediately from Theorem 1 and the following proposition which was kindly communicated to us by Professor Eilenberg.

Proposition 3. Let $F$ be a field, $A$ an $F$-algebra, and $A_{1} \subset A_{2} \subset \cdots$ $\subset A_{i} \subset \cdots$ a sequence of subalgebras of $A$ such that $A=\cup_{1}^{\infty} A_{i}$. Then

$$
\operatorname{dim} A \leqq 1+\sup _{i} \operatorname{dim} A_{i}
$$

Proof. Assume $\operatorname{dim} A_{i}<n$ for all $i$, and let $f$ be an $(n+1)$-cocycle of $A$ into a two-sided $A$-module, $N$. We shall define by induction a sequence of $n$-cochains $g_{i}$ from $A_{i}$ to $N$ such that

$$
g_{i+1} \mid A_{i}=g_{i} \text { and } \delta g_{i}=f \mid A_{i} .
$$

Assume $g_{i}$ already defined. Since $H^{n+1}\left(A_{i+1}, N\right)=0$ there is a cochain $h$ on $A_{i+1}$ such that

$$
\delta h=f \mid A_{i+1} .
$$

Then $\delta g_{i}=(\delta h) \mid A_{i}$ and thus $g_{i}-h \mid A_{i}$ is an $n$-cocycle on $A_{i}$. Since $H^{n}\left(A_{i}, N\right)$ $=0$ there is an $(n-1)$-cochain $k$ on $A_{i}$ such that

$$
\delta k=g_{i}-h \mid A_{i} .
$$

Let $k^{\prime}$ be an arbitrary extension of the cochain $k$ to $A_{i+1}$, i.e., $k^{\prime} \mid A_{i}=k$. Such an extension exists since $A_{i}$ is an $F$-direct summand of $A_{i+1}$. If we set

$$
g_{i+1}=h+\delta k^{\prime}
$$

then

$$
g_{i+1}\left|A_{i}=h\right| A_{i}+\delta k=g_{i}, \quad \delta g_{i+1}=\delta h=f \mid A_{i+1}, \text { as desired. }
$$

(5) Every finite subset can be embedded in a separable subalgebra of finite order. 
The sequence of cochains $\left\{g_{i}\right\}$ defines a single cochain $g$ on $A$ such that $\delta g=f$. Thus $H^{n+1}(A, N)=0$ and $\operatorname{dim} A \leqq n$.

4. Additivity of dimension. We begin this section by reading down two of the "change of rings" theorems of [4].

Proposition 4 [4, VI, 4.1.4]. Let $R$ be a ring with subring $S$ such that $R$ is projective as a left $S$-module. Then for any $R$-module $M, 1 \cdot \operatorname{dim}_{R} M \geqq 1 \cdot \operatorname{dim}_{S} M$.

Proposition 5 [4, VI, 4.1.3]. Let $R$ be any ring with subring $S$ such that $R$ is projective as a right $S$-module. Then for any $S$-module $M$,

$$
\operatorname{l.dim}_{S} M \geqq 1 \cdot \operatorname{dim}_{R} R \otimes_{S} M \text {. }
$$

We shall also need the following proposition which may be verified by direct computation.

Proposition 6. When $f$ is a function of $n+1$ variables $u_{1}, \cdots, u_{n+1}$, denote by $\mathfrak{A}_{n+1} f$ the function

$$
\mathfrak{A}_{n+1} f\left(x_{1}, \cdots, x_{n+1}\right)=\sum(\operatorname{sgn} \pi) f\left(x_{\pi(1)}, \cdots, x_{\pi(n+1)}\right)
$$

the sum ranging over all permutations $\pi$ of $\{1, \cdots, n+1\}$. If $g$ is an $n$-cochain on a commutative algebra then $\left({ }^{6}\right)$.

$$
\begin{aligned}
& \mathfrak{A}_{n+1} \delta g\left(x_{1}, \cdots, x_{n+1}\right) \\
& \quad=\sum_{i=1}^{n+1}(-1)^{i-1}\left[x_{i} \mathfrak{A}_{n} g\left(x_{1}, \cdots, \hat{x}_{i}, \cdots, x_{n}\right)-\mathfrak{A}_{n} g\left(x_{1}, \cdots, \hat{x}_{i}, \cdots, x_{n}\right) x_{i}\right] .
\end{aligned}
$$

We now prove the main theorem of this section.

THEOREM 5. Let $A$ be an algebra over the commutative ring $K$ such that $A$ is a free $K$-module (this is automatic if $K$ is a field), and let $F$ be a subring of $K$. If $F-\operatorname{dim} K=n$ and $K-\operatorname{dim} A=p$, then $n \leqq F-\operatorname{dim} A \leqq n+p$.

Proof. Since $A$ is $K$-free, $K \cong K \cdot 1$ is in the center of $A$. Therefore, $A$ is a direct sum of copies of $K$ even as a $K^{e}$-module so that by [4, VI, 1.2] $\operatorname{lddim}_{K^{e}} A$ $=\operatorname{ldim}_{K e} K=n$. Now $A^{e}$ is also a direct sum of copies of $K^{e}$. Thus Proposition 4 yields $F$-dim $A=1 \cdot \operatorname{dim}_{A} e A \geqq 1 \cdot \operatorname{dim}_{K^{e}} A=n$.

Similarly by Proposition $5,1 \cdot \operatorname{dim}_{K^{e}} K \geqq \operatorname{ldim}_{A} e A^{e} \otimes_{K} K$. But we have the following natural $A^{e}$-isomorphisms: $A^{e} \otimes_{K e} K=\left(A_{8} \otimes A_{d}\right) \otimes_{K} \otimes K K$ $\cong A_{s} \otimes_{F \otimes K}\left(A_{d} \otimes_{K} K\right) \cong A_{8} \otimes_{K} A_{d}$, where the isomorphism between the second and third terms is given by $\left(a \otimes a^{*}\right) \otimes k \rightarrow a \otimes\left(a^{*} \otimes k\right)$. We omit the straightforward proof (cf. [4, IX, 2.1]). Thus $\operatorname{ldim}_{A^{e}} A_{s} \otimes_{K} A_{d} \leqq n$. Since $p=1 . \operatorname{dim}_{A \otimes_{K^{A}}} A$ and $A \otimes_{K} A^{*}$ is a homomorph of $A^{e}$, [7, Proposition 3] is available and asserts $\operatorname{ldim}_{A} e A \leqq n+p$, proving the theorem.

To show that $F$-dim $A$ may very well be less than $n+p$, we offer the following two examples.

(6) $\hat{x}_{i}$, means that $x_{i}$ is to be omitted. 
(i) Let $A$ be a separable algebraic extension field of a field $F$ with $(A: F)$ $=\boldsymbol{\aleph}_{0}$. Then there is a subfield $K$ of $A$ such that $(K: F)=(A: K)=\boldsymbol{\aleph}_{0}$ also. Theorem 4 then yields $F$-dim $A=K$-dim $A=F-\operatorname{dim} K=1$.

(ii) Let $F$ be a field of characteristic $p \neq 0, t$ an indeterminate over $F, K=F(t)$, and $A=K\left(t^{1 / p}\right)$. Then Theorem 7 below shows that $F$-dim $K=1$ and by $\left[10\right.$, p. 946] or our 'Theorem $9, K-\operatorname{dim} A=\infty$. However, $A=F\left(t^{1 / p}\right)$ is merely a rational function field over $F$ and so again by Theorem $7, F$-dim $A$ $=1$.

Examples where the dimension is additive are given by the following two theorems.

Theorem $6[4, \mathrm{IX}, 7.11]$. Let $F$ be a commutative ring, and $A$ be the polynomial ring $F\left[t_{1}, \cdots, t_{n}\right]$ in $n$ independent indeterminates over $F$. Then $F-\operatorname{dim} A=n$.

Proof. First, $\operatorname{dim} A \geqq n$ because we can exhibit a nonzero element of $H^{n}(A, A)$ : with $u_{1}, \cdots, u_{n}$ varying in $A$, let

$$
f\left(u_{1}, \cdots, u_{n}\right)=\prod_{i=1}^{n} \frac{\partial u_{i}}{\partial t_{i}}
$$

where $\partial u_{i} / \partial t_{i}$ denotes the ordinary partial derivative of the polynomial $u_{i}$. Routine calculation shows that this is an $n$-cocycle of $A$ into $A$. Since $A$ is commutative, any coboundary $\delta g$ of $A$ into $A$ satisfies $\mathfrak{A}_{n} \delta g\left(u_{1}, \cdots, u_{n}\right)=0$ by Proposition 6. But direct evaluation gives $\mathfrak{A}_{n} f\left(t_{1}, \cdots, t_{n}\right)=1$, so that $f$ is not a coboundary. (Essentially the same proof that $f$ is not a coboundary is already to be found in [5].)

Next we prove $\operatorname{dim} A \leqq n$ by induction on $n$. If $n=1, A=F[t], A^{e}=A \otimes A^{*}$ $=F\left[t^{\prime}, t^{\prime \prime}\right]$, where $t^{\prime}=t \otimes 1$ and $t^{\prime \prime}=1 \otimes t$ are independent indeterminates over $F$. The natural ring-homomorphism of $A^{*}=A \otimes A^{*}$ onto $A=A \otimes{ }_{A} A^{*}$ has as kernel the principal ideal generated by $t^{\prime}-t^{\prime \prime}$ since $p\left(t^{\prime}, t^{\prime \prime}\right)$ is in the kernel if and only if $p(t, t)=0$. But the only element in $A^{e}$ annihilating $t^{\prime}-t^{\prime \prime}$ is 0 so this kernel is a free $A^{e}$-module and

$$
0 \rightarrow\left(t^{\prime}-t^{\prime \prime}\right) A^{e} \rightarrow A^{e} \rightarrow A \rightarrow 0
$$

is a projective resolution for $A$ over $A^{e}$. Thus $\operatorname{dim} A \leqq 1$. Now assume the inequality true for the algebra $K=F\left[t_{1}, \cdots, t_{n-1}\right]$ and consider $A=K\left[t_{n}\right]$. By the case $n=1, K-\operatorname{dim} A \leqq 1$ and by the induction hypothesis, $F-\operatorname{dim} K$ $\leqq n-1$. Hence Theorem 5 proves $F$-dim $A \leqq n$.

THEOREM 7. Let $F$ be a field and $A$ the field of rational functions

$$
F\left(t_{1}, \cdots, t_{n}\right)
$$

in $n$ independent indeterminates over $F$. Then $F-\operatorname{dim} A=n$.

The proof is identical with that of Theorem 6 once we note that when 
$n=1, A^{e}$ is the integral domain consisting of all rational functions

$$
p\left(t^{\prime}, t^{\prime \prime}\right) / q\left(t^{\prime}\right) r\left(t^{\prime \prime}\right)
$$

with $p, q, r$ polynomials over $F$.

Besides Theorem 6, Cartan and Eilenberg also prove that if $F$ is semisimple the global dimension of the polynomial ring $A$ is $n$. Both these facts are proved in [4] by explicit construction of a projective resolution for $F$ as an $A$-module. We shall now give an alternative proof of this result on $1 . g l . d i m ~ A$ which reduces the problem to one of algebra dimension and avoids this projective resolution.

If $F$ is semisimple, 1.gl.dim $A \leqq \operatorname{dim} A[4, \mathrm{IX}, 7.6]$. Since $\operatorname{dim} A=n$ by Theorem 6 , it is sufficient to exhibit a left $A$-module with left $A$-dimension at least $n$. We turn $F$ into an $A$-module by setting $t_{i} F=0$. Now, $A^{e}$ is the ring of polynomials in $2 n$ indeterminates $t_{i}^{\prime}, t_{i}^{\prime \prime}(i=1, \cdots, n)$ with $t_{i}^{\prime}=t_{i} \otimes 1$ and $t_{i}^{\prime \prime}=1 \otimes t_{i}$. We make it an $A$-module by identifying $A$ with the subring $A_{0}=F\left[t_{1}^{\prime}-t_{1}^{\prime \prime}, \cdots, t_{n}^{\prime}-t_{n}^{\prime \prime}\right]$ of $A^{e}$. Then $A^{e}$ is a ring of polynomials in $n$ indeterminates over $A_{0}$ and so is a free $A_{0}$-(hence $A$-) module. Proposition 5 then gives $1 . \operatorname{dim}_{A} F \geqq 1 . \operatorname{dim}_{A^{e}} A^{e} \otimes_{A} F$. But $A^{e} \otimes_{A} F \cong A$ as $A^{e}$-modules, the isomorphism being given by $b \otimes f \rightarrow b f$ where $b \rightarrow b$ is the natural mapping of $A^{6}$ onto $A\left({ }^{7}\right)$. We omit the straightforward verification that this is an $A^{e_{-}}$isomorphism. Thus $1 \cdot \operatorname{dim}_{A} F \geqq 1 \cdot \operatorname{dim}_{A} 0 A=\operatorname{dim} A=n$ by Theorem 6 , which proves l.gl.dim $A \geqq n$.

5. Commutative algebras with minimum condition. If $A$ is a commutative algebra over a field $F$ satisfying the minimum condition, $\operatorname{dim} A=\infty$ if $A$ is not semisimple ([3, Proposition 15] and [4, IX, 7.6]). Thus we can restrict ourselves to the semisimple case where $A$ is a direct sum of fields. By $[4, \mathrm{IX}, 7.3]$ we may then restrict ourselves to the case where $A$ is itself a field.

Theorem 8. If $A$ is a field of transcendence degree $n \leqq \infty$ over the field $F$, then $\operatorname{dim} A \geqq n$.

Proof. For any finite $m \leqq n, A$ contains a rational function field $K$ in $m$ indeterminates. Theorems 5 and 7 then show $m \leqq \operatorname{dim} A$.

THEOREM 9. If $A$ is a finitely generated extension field of $F$ with no separable generation over $F$, then $\operatorname{dim} A=\infty$.

Proof. Of course the base field $F$ has a characteristic $p \neq 0$. Let $A=F\left(x_{1}\right.$, $\left.\cdots, x_{r}\right)$ and let $s$ be the largest integer $\geqq 0$ such that $S=F\left(x_{1}, \cdots, x_{s}\right)$ can be separably generated over $F$. Let $\left\{t_{1}, \cdots, t_{n}\right\}$ be a transcendence

(7) The tempting string of natural isomorphisms (as in Theorem 5) $A^{\bullet} \otimes_{\Lambda} F=\left(A_{\bullet} \otimes A_{d}\right)$ $\otimes_{A} F \cong A_{\bullet} \otimes\left(A_{d} \otimes_{A} F\right) \cong A_{d} \otimes_{F} F \cong A_{\text {s }}$ will not do here. It can be arranged to give a group iso-

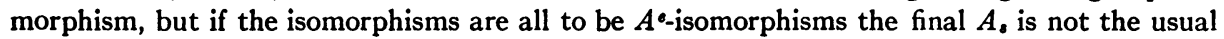
$A^{\bullet}$-module $A$ but rather is annihilated by multiplication by $1 \otimes i$. 
basis of $S$ such that $S$ is separable over the rational function field $K=F\left(t_{1}, \cdots, t_{n}\right)$, and write $y=x_{8+1}$. Then we assert that $K(y)$ has no separable generation over $F$. For suppose otherwise. Since $S$ is separable over $K$, $S(y)$ is separable over $K(y)$, whence $F\left(x_{1}, \cdots, x_{8}, x_{s+1}\right)=S(y)$ is separably generated over $F$, contradicting the choice of $s$. By Theorem 5 , it suffices to prove $\operatorname{dim} K(y)=\infty$.

Let $\phi$ be an irreducible polynomial in $n+1$ indeterminates over $F$ such that

$$
\phi\left(t_{1}, \cdots, t_{n}, y\right)=0 .
$$

Since $y$ is inseparable over $K$ and (5) is the minimum equation of $y$ over $K$, in every nonzero term of $\phi$ the exponent of $y$ is a multiple of $p$. If $t_{i}$ actually occurs in (5) then $\left\{t_{1}, \cdots, t_{i}, \cdots, t_{n}, y\right\}\left(^{6}\right)$ is another transcendence basis of $K(y)$ over $F$. Since $K(y)$ has no separable generation over $F, t_{i}$ plays the same role with respect to $F\left(t_{1}, \cdots, \hat{t}_{i}, \cdots, t_{n}, y\right)$ as $y$ does with respect to $K$, and the exponents of $t_{i}$ in (5) are also multiples of $p$.

Now let $G$ be the field obtained by adjoining to $F$ the $p$ th roots of the coefficients of $\phi$ so that $\phi$ becomes the pth power of a polynomial $\phi^{1 / p}$ with coefficients in $G$. Let $z=\phi^{1 / p}\left(t_{1}, \cdots, t_{n}, y\right) \in G \otimes K(y)$ so that $z^{p}=0$. On the other hand, $z \neq 0$, for if a collection of products of powers of $t_{1}, \cdots, t_{n}, y$ (as in the terms of $\phi^{1 / p}$ ) involve no higher power of $y$ than appears in $\phi\left(t_{1}, \cdots, t_{n}, y\right)$, then these power products are linearly independent in $K(y)$ over $F$, hence also in $G \otimes K(y)$ over $G$. Therefore $G \otimes K(y)$ is a commutative algebra over $G$ satisfying the minimum condition [2, Theorem $6.10 \mathrm{~B}$ ] and containing a nilpotent ideal. By the first sentence in this section,

$$
G \text {-dim }(G \otimes K(y))=\infty .
$$

Since the dimension of an algebra is invariant under change of base field [4, IX, 7.2 ], $F$-dim $K(y)=\infty$ also.

We note that Theorem 9 also yields Hochschild's result that a semisimple inseparable algebra of finite order has dimension $\infty[10$, p. 946]. Clearly it is sufficient to treat the case of a simple algebra $A$ whose center $Z$ is an inseparable finite extension field of $F$. Then by Theorem $9, \operatorname{dim} Z=\infty$ and since $A$ is $Z$-free, Theorem 5 gives $\operatorname{dim} A=\infty$ also.

THEOREM 10. If $A$ is a field of transcendence degree $n<\infty$ over a field $F$, then $\operatorname{dim} A=n$ if and only if $A$ is finitely, separably generated over $F$ (i.e., $A$ is a finite, separable, algebraic extension of a rational function field in $n$ indeterminates).

Proof. If $A$ is a finite, separable, algebraic extension of a rational function field, $K$, then $K-\operatorname{dim} A=0$ [8, Theorem 4.1] and $F-\operatorname{dim} K=n$ by Theorem 7 . The sufficiency follows from Theorem 5 . Theorem 9 yields one part of the necessity proof. It remains to show that if $A$ is an infinite algebraic extension 
of a rational function field $K=F\left(t_{1}, \cdots, t_{n}\right)$ then $\operatorname{dim} A>n$. First, $A$ contains a subfield $A^{\prime}$ with $\left(A^{\prime}: K\right)=\boldsymbol{N}_{0}$ and by Theorem 5 it is sufficient to prove that $\operatorname{dim} A^{\prime}>n$. So, without loss of generality, we may assume that $(A: K)=\aleph_{0}$. Theorem 3 guarantees a $K$-linear derivation $\Delta$ of $A$ into $A_{8} \otimes_{K} A_{d}$ which is not inner. Also $K$ has $n$ derivations $\Delta_{i}=\partial / \partial t_{i}$ into itself satisfying $\Delta_{i}\left(t_{j}\right)=0$ for $i \neq j$ and $\Delta_{i}\left(t_{i}\right)=1$. These $\Delta_{i}$ can be extended to derivations of $A$ thus: In terms of a basis $\left({ }^{8}\right)$ of $A$ over $K$ left multiplication by an element $a$ in $A$ may be represented as a column-finite matrix $\mu$ with entries in $K$. Define $\Delta_{i}(a)$ as the matrix obtained by applying $\Delta_{i}$ to all the entries in $\mu$. This gives $n$ derivations of $A$ into the ring of all $K$-linear transformations on $A$, which is naturally isomorphic to the ring of all $\left(1 \otimes_{K} A\right)$-linear transformations on $A_{8} \otimes_{K} A_{d}$. Since the elements of $K$ are represented by scalar matrices, we still have $\Delta_{i}\left(t_{j}\right)=0$ for $i \neq j$ and $\Delta_{i}\left(t_{i}\right)=1$. Then construct

$$
f\left(u_{1}, \cdots, u_{n+1}\right)=\Delta_{1}\left(u_{1}\right) \Delta_{2}\left(u_{2}\right) \cdots \Delta_{n}\left(u_{n}\right) \Delta\left(u_{n+1}\right) .
$$

Direct computation shows this is an $(n+1)$-cocycle of $A$ into $A_{,} \otimes_{K} A_{d}$. Suppose $f=\delta g$ and use the identity in Proposition 6 with $u_{i}=t_{i}(i=1, \cdots, n)$ and $u_{n+1}$ arbitrary. The left side of the identity becomes $\Delta\left(u_{n+1}\right)$ because $\Delta_{i}\left(t_{j}\right)=0$ for $j \neq i$. Since $u_{i}=t_{i} \in K, u_{i} x-x u_{i}=0$ for $x \in A_{,} \otimes_{K} A_{d}$ and the right side of the identity becomes $u_{n+1} y-y u_{n+1}$ with $y=\mathfrak{A}_{n} g\left(t_{1}, \cdots, t_{n}\right)$. This means $\Delta$ is inner, contrary to construction (Theorem 3). Thus $f \operatorname{deter-}$ mines a nonzero element of $H^{n+1}\left(A, A_{8} \otimes_{K} A_{d}\right)$, proving Theorem 10.

If $A$ is separable over $K$, the proof can avoid the assumption $(A: K)=\boldsymbol{\aleph}_{0}$, the use of Theorem 3 and the matrix arguments: extend the partial derivatives $\Delta_{i}$ on $K$ in the usual way to derivations of $A$ into $A$. Let $\Delta$ be any noncobounding 1-cocycle of $A$ over $K$ into an $\left(A \otimes_{K} A^{*}\right)$-module $N$ ( $\Delta$ exists by Theorem 1). Then construct the noncobounding $(n+1)$-cocycle $f$ of $A$ into $N$ by the formula (6).

In case $(A: K)=\boldsymbol{\aleph}_{0}$ we can actually determine $\operatorname{dim} A^{\prime}$ completely:

Corollary. Let $A$ be an extension field of transcendence degree $n$ over $F$ which is countably generated but not finitely generated. Then $\operatorname{dim} A=\infty$ or $n+1$ according as $A$ is not or is locally separably generated $\left({ }^{3}\right)$.

Proof. If $A$ is not locally separably generated, Theorem 9 and Theorem 5 assert $\operatorname{dim} A=\infty$. (This of course does not need the countable generation.) Otherwise $A=\cup_{1}^{\infty} A_{i}$ where each $A_{i}$ is a finitely separably generated extension of $F$ and so has dimension $\leqq n$ by Theorem 10. Proposition 3 then shows that $\operatorname{dim} A \leqq n+1$ and Theorem 10 gives $\operatorname{dim} A \geqq n+1$.

(8) This extension of derivations need not depend on a choice of basis or a matrix representation: Indeed, in a forthcoming paper we shall show that if $L$ is the ring of all linear transformations on a vector space over a division ring $K$, and $F$ is a subfield of the center of $K$, any derivation over $F$ of $K$ into $K$ has an extension to a derivation of $L$ into $L$; this extension is unique up to an inner derivation. Moreover, $H^{n}(L, L) \cong H^{n}(K, K)$. 
We note that $A$ may be locally separably generated without being separably generated itself: let $F$ be a perfect field of characteristic $p \neq 0$ and let $A=F\left(t, t^{p^{-1}}, t^{p^{-2}}, \cdots, t^{p^{-n}}, \cdots\right)$. Then $A=\bigcup F\left(t^{p^{-n}}\right)$ but $A$ is not separably generated. Indeed, if it were it would admit nonzero derivations into itself, but since every element of $A$ is a $p$ th power this is impossible.

Finally we remark that the proof of Theorem 10 even gives some information about noncommutative algebras $A$. In particular if $A$ contains in its center a rational function field $K$ in $n$ indeterminates over $F$ and if

$$
H^{1}\left(A, A_{s} \otimes_{K} A_{d}\right) \neq 0
$$

(cf. Theorems 2 and 3) then $F$-dim $A>n$. If in addition $(A: K)=\boldsymbol{N}_{0}$ and $A$ is locally separable over $K$, then $F$-dim $A=n+1$-which, when $n=0$, does not quite include Theorem 4.

\section{REFERENCES}

1. A. A. Albert, Structure of algebras, New York, 1939.

2. E. Artin, C. Nesbitt, and R. Thrall, Rings with minimum condition, Ann Arbor, 1944.

3. M. Auslander, On the dimension of modules and algebras III, Nagoya Math. J. vol. 9 (1955) pp. 67-77.

4. H. Cartan and S. Eilenberg, Homological algebra, Princeton, 1956.

5. W. C. Carter, On the cohomology theory of fields, unpublished doctoral dissertation, Harvard University, 1947.

6. S. Eilenberg, Algebras of cohomologically finite dimension, Comment. Math. Helv. vol. 28 (1954) pp. 310-319.

7. S. Eilenberg, M. Ikeda, and T. Nakayama, On the dimension of modules and algebras I, Nagoya Math. J. vol. 8 (1955) pp. 49-57.

8. G. Hochschild, On the cohomology groups of an associative algebra, Ann. of Math. vol. 46 (1945) pp. 58-67.

9. On the cohomology theory for associative algebras, Ann. of Math. vol. 47 (1946) pp. 568-579.

10. - Cohomology and representation of associative algebras, Duke Math. J. vol. 14 (1947) pp. 921-948.

11. M. Ikeda, H. Nagao, and T. Nakayama, Algebras with vanishing n-cohomology groups, Nagoya Math. J. vol. 7 (1954) pp. 115-131.

12. N. Jacobson, The theory of rings, New York, 1943.

13. V. M. Kurockin, The decomposition of algebras into a semi-direct sum of the radical and a semisimple subalgebra, C. R. (Doklady) Acad. Sci. URSS (N. S.) vol. 36 (1942) pp. 42-45.

14. D. Zelinsky, Raising idempotents, Duke Math. J. vol. 21 (1954) pp. 315-322.

NORTHWESTERN UNIVERSITY

Evanston, ILl. 